\title{
INESTABILIDAD, CRECIMIENTO Y DESEMPEÑO ECONÓMICO: EVIDENCIA DE 17 PAÍSES DE AMÉRICA LATINA, 1980-2014*
}

\author{
DOI: DOI: $10.22395 /$ seec.v19n41a5 \\ Carlos D. Dabús** \\ Fernando Delbianco ${ }^{* * *}$ \\ Pablo D. Monterubbianesi ${ }^{* * * *}$
}

Recibido: 06 de junio de 2016 • Aprobado: 05 de septiembre de 2016

\section{RESUMEN}

El trabajo estudia el efecto de la inestabilidad económica sobre el crecimiento en 17 países de Latinoamérica en el período 1980-2014 para tres diferentes grupos de crecimiento económico: bajo, medio y alto. La hipótesis es que una economía más inestable, es decir, con mayor inflación y volatilidad del producto perjudica a la inversión y, por tanto, al crecimiento. Para contrastar esta hipótesis se estima un modelo de efectos aleatorios usando un panel de 17 países de América Latina de un período de 35 años, en el cual se clasifican los países de acuerdo con el método de clúster de k-medianas. Los resultados indican que la inestabilidad afecta negativamente el desempeño económico de la región, en particular en los países de menor crecimiento. Por tanto, en estos casos se recomienda la aplicación de políticas anticíclicas y anti-inflacionarias.

\section{PALABRAS CLAVE}

Ciclos económico, crecimiento económico, políticas anti-cíclicas, América Latina

\section{CLASIFICACIÓN JEL}

O40, E30, E60, O54

\section{CONTENIDO}

Introducción; 1. Revisión de la literatura; 2. Datos y metodología; 3. Evidencia empírica; 4. Conclusiones; Bibliografía.

Artículo de investigación desarrollado en el marco del proyecto "Factores internos y externos del crecimiento en Latinoamérica", con vigencia durante el periodo 2013-2016, financiado por la Secretaría de Ciencia y Tecnología de la Universidad Nacional del Sur.

** Licenciado en Economía, Universidad Nacional del Sur, Bahía Blanca, Argentina. Magíster en Economía, Instituto Torcuato Di Tella, Buenos Aires, Argentina. Doctor en Economía, Universidad Nacional del Sur, Bahía Blanca, Argentina. Profesor titular del Departamento de Economía, Universidad Nacional del Sur e Investigador Principal del CONICET, Bahía Blanca, Argentina. Dirección: Departamento de Economía, Universidad Nacional del Sur, San Andrés 800, Altos de Palihue, 8000, Bahía Blanca, Argentina. Teléfono: +54-291-4595138 - interno 2720. Correo electrónico: cdabus@criba.edu.ar.

** Licenciado en Economía, Universidad Nacional del Sur, Bahía Blanca, Argentina. Máster en Econometría, Universidad di Tella, Buenos Aires, Argentina. Doctor en Economía, Universidad Nacional del Sur, Bahía Blanca, Argentina. Asistente de Docencia del Departamento de Economía de la Universidad Nacional del Sur, Bahía Blanca, Argentina. Dirección: Departamento de Economía, Universidad Nacional del Sur, San Andrés 800, Altos de Palihue, 8000, Bahía Blanca, Argentina. Teléfono: +54-291-4595138 - interno 2712. Correo electrónico: fernando.delbianco@uns.edu.ar.

*** Licenciado en Economía, Universidad Nacional del Sur, Bahía Blanca, Argentina. Doctor en Economía, Universidad Nacional del Sur, Bahía Blanca, Argentina. Asistente de Docencia del Departamento de Economía de la Universidad Nacional del Sur e Investigador Asistente del CONICET, Bahía Blanca, Argentina. Dirección: Departamento de Economía, Universidad Nacional del Sur, San Andrés 800, Altos de Palihue, 8000, Bahía Blanca, Argentina. Teléfono: +54-291-4595138 - interno 2716. Correo electrónico: pmonteru@uns.edu.ar. 


\section{INSTABILITY, GROWTH AND ECONOMIC PERFORMANCE: EVIDENCE OF 17 LATIN AMERICAN COUNTRIES 1980-2014}

\section{ABSTRACT}

This paper studies the effect of economic stability on the growth of 17 Latin American countries between 1980-2014 for three different economic growth groups; low, middle and high. The hypothesis is that a more unstable economy, meaning, a larger inflation and product volatility, negatively affects investments, which affects growth. In order to contrast this hypothesis, a random effect model is estimated using a panel of 17 Latin American countries in a 35-year period, in which countries are classified according to the k-median cluster method. Results indicate that instability affects negatively the economic performance of the region, particularly countries with a smaller growth. Because of this, the recommendation for such cases is the application of anti-cyclic and anti-inflationary policies.

\section{KEY WORDS}

Economic cycles, economic growth, anti-cyclic policies, Latin America.

\section{JEL CLASSIFICATION}

O40, E30, E60, O54

\section{CONTENIDO}

Introduction; 1. Literature review; 2. Data and methodology; 3. Empiric evidence; 4. Conclusions; Bibliography.

\section{INSTABILIDADE, CRESCIMENTO E DESEMPENHO ECONÔMICO: EVIDÊNCIA DE 17 PAÍSES DA AMÉRICA LATINA, 1980-201}

\section{RESUMO}

O trabalho estuda o efeito da instabilidade econômica sobre o crescimento em 17 países da Latino-américa no período 1980-2014 para três diferentes grupos de crescimento econômico: baixo, médio e alto. a hipótese é que uma economia mais instável, ou seja com maior inflação e volatilidade do produto, prejudica ao investimento e por tanto ao crescimento. Para contrastar esta hipótese se estima um modelo de efeitos aleatórios usando um painel de 17 países da América Latina de um período de 35 anos, no qual se classificam os países de acordo ao método de clúster de k-medianas. Os resultados indicam que a instabilidade afeta negativamente o desempenho econômico da região, em particular nos países de menor crescimento. Por tanto, nestes casos se recomenda a aplicação de políticas anticíclicas e anti-inflacionárias.

\section{PALAVRAS CHAVE}

Ciclos econômico, crescimento econômico, políticas anticíclicas, América Latina

\section{CLASSIFICAÇÃO JEL}

O40, E30, E60, O54

\section{CONTEÚDO}

Introdução; 1. Revisão da literatura; 2. Dados e metodologia; 3. Evidência empírica; 4. Conclusões; Bibliografia. 


\section{INTRODUCCIÓN}

Existe un amplio conjunto de factores que pueden ser relevantes para explicar los procesos de crecimiento y estancamiento económico en América Latina. Asimismo, la variedad de resultados encontrados indica la complejidad del fenómeno. La literatura, en tal sentido, muestra evidencia sobre los determinantes del crecimiento en la región, como, por ejemplo, las contribuciones de Pardo y Reig (2002) para Uruguay. Los autores encuentran que la demanda de exportaciones resulta un factor clave para explicar el proceso de evolución del ingreso en dicha economía, poniéndose en evidencia la restricción que genera la balanza de pagos. Brizeño, Cercone y Cardoza (2003) abordan la temática para Costa Rica, y encuentran que la inversión en capital físico y humano favorece el crecimiento. A su vez, Clavijo (2003) y Durán y Hurtado (2009) analizan el caso de Colombia. En el primer caso, el autor encuentra que la inversión y el grado de apertura favorecen al proceso de crecimiento en el largo plazo. Mientras tanto, en el segundo caso los autores destacan que los aspectos internacionales tales como la liquidez mundial, los términos de intercambio y el dinamismo de los patrones del comercio juegan un rol fundamental en dicho proceso.

Por su parte, Coremberg (2006), en un análisis para Argentina durante el período 1990-2004, destaca el rol de las ganancias de competitividad como fuente relevante del crecimiento. Asimismo, el autor resalta que la economía argentina debe orientar sus políticas hacia el aumento de la productividad, si quiere lograr un proceso de crecimiento sostenido en el largo plazo. Por último, Cimoli et al. (2008) para el caso de Brasil encuentran que los cambios en la estructura productiva, en particular los incrementos en la participación de sectores intensivos en tecnología, son un determinante clave para el crecimiento.

Por otro lado, hay un posible nexo entre desigualdad y crecimiento económico, el cual ha sido discutido en la literatura. Desde el trabajo precursor de Kuznets (1955), y la publicación de la base de datos de desigualdad hecha por Deininger y Squire (1996), hubo un cuerpo creciente de evidencia respecto al efecto de la desigualdad del ingreso sobre el crecimiento económico. La evidencia muestra resultados mixtos, en términos tanto teóricos como empíricos; por ejemplo, para evidencia empírica eclética en América Latina sobre tal relación ver Delbianco, Dabús y Caraballo (2014).

En síntesis, existe un amplio conjunto de factores que pueden ser relevantes para explicar los períodos de crecimiento y estancamiento en Latinoamérica. No obstante, es de destacar que la región está comprendida por países similares, en temas tales como el de constituir un bloque de economías en vías de desarrollo, con una historia de baja calidad institucional y política, y con un mercado interno pequeño. 
En particular, el rasgo fundamental de los países latinoamericanos parece haber sido el de la inestabilidad económica. En este sentido, Bermúdez, Dabús y González (2015), en un estudio regional para el período 1950-2009, encuentran que los períodos tanto de alta inflación como de volatilidad del producto han sido perjudiciales para el desempeño económico. Más aún, su efecto se ha mostrado robusto a diferentes especificaciones del análisis empírico, mientras que la calidad institucional y la estabilidad política no han sido relevantes para explicar el crecimiento.

A su vez, la región en el tiempo ha tenido períodos en que disfrutó de mayor crecimiento, así como otros de severo estancamiento; por ejemplo, ver Gutiérrez (1999) para una discusión sobre el crecimiento en la segunda mitad de la década de 1990 de las principales economías de América Latina, y Dabús, González y Bermúdez (2012) para una descripción de la evolución del crecimiento a largo plazo en la región. Por tanto, la evidencia parece indicar que sería interesante profundizar el análisis de la influencia de la inestabilidad económica sobre el crecimiento en Latinoamérica. Por tal motivo, el objetivo de este trabajo consiste en analizar dicha influencia en las economías de la región en el período 1980-2014; el estudio se realiza para tres diferentes regímenes de crecimiento': bajo, medio y alto. A tal fin, se aplica un análisis de clúster de medianas a la muestra total de países para la obtención de dichos regímenes. La hipótesis es que una economía más inestable, es decir, con mayor inflación y volatilidad del producto perjudica a la inversión y, por tanto, al crecimiento.

Precisamente, el aporte del trabajo consiste en analizar el efecto de la inestabilidad sobre el desempeño económico en diferentes regímenes de crecimiento, los que se obtienen por medio de una técnica de clústeres de medianas. Esto evita la influencia de los valores extremos al momento en la obtención de dichos regímenes. A su vez, a diferencia de la literatura tradicional sobre el crecimiento, el hecho de que aquí el estudio del efecto de la inestabilidad sobre el desempeño económico se hace en el contexto de diferentes regímenes permite identificar ciertas particularidades de la inestabilidad, especialmente, si dicha inestabilidad es o no igual de perjudicial en períodos de fuerte crecimiento que en períodos de estancamiento. En particular, se espera que el efecto de la inestabilidad sobre el desempeño económico sea más perjudicial en períodos de menor crecimiento, lo que efectivamente corrobora la evidencia empírica aquí encontrada, mostrada más adelante.

El análisis empírico se realiza mediante datos de panel; a partir de las características de los datos se estima un modelo de efectos aleatorios. Por un lado, se

Se entiende como régimen de crecimiento a un período de tiempo en el cual la tasa de crecimiento del producto per cápita se mantiene en un rango de valores similares. 
incluyen como variables explicativas del mismo las que en forma tradicional se han utilizado como variables de control, es decir las consideradas como robustas por Levine y Renelt (1992): la tasa de inversión física sobre el producto, la tasa de inversión en capital humano, la tasa de crecimiento de la población y el coeficiente de apertura. A su vez, en función de la literatura antes mencionada, a estas se agregan como variables explicativas la desigualdad, tomada a partir del coeficiente de Gini, y en particular la inestabilidad económica. Esta última, de acuerdo con el trabajo de Bermúdez, Dabús y González (2015), es aproximada por la volatilidad de la tasa de crecimiento del producto (calculada como la desviación estándar de la misma), y la tasa de inflación.

El trabajo está organizado como sigue. En la sección siguiente se realiza una revisión de la literatura sobre crecimiento en Latinoamérica. En la sección 2 se presentan los datos y la metodología utilizada en el análisis empírico. En la sección 3 se estima el efecto de la inestabilidad sobre el crecimiento, tanto para la muestra global de países como para los diferentes regímenes de crecimiento. Por último, la sección 4 presenta las conclusiones.

\section{REVISIÓN DE LA LITERATURA}

En general los estudios del crecimiento en América Latina en términos regionales se pueden agrupar en cuatro categorías. En primer lugar están los que enfatizan el rol de los factores acumulables (el capital físico y el capital humano) en el crecimiento. Entre estos se encuentra Astorga (2010), quien realiza un estudio de largo plazo de crecimiento en la región. Por el contrario, en el segundo grupo de trabajos están los que señalan que la productividad total de los factores (PTF) es la variable clave para explicar el crecimiento como Manuelli (2005) y Chumacero y Fuentes (2006), entre otros. Asimismo, Singh y Cerisola (2006) encuentran resultados empíricos que se ubican en ambas categorías: según su evidencia las economías de más rápido crecimiento han estado asociadas a una mayor PTF y acumulación de capital, las que, a su vez, fueron favorecidas por la aplicación de políticas macroeconómicas estables.

En tercer lugar están los aportes que destacan la importancia de la estabilidad económica y política como factores explicativos del crecimiento. Entre ellos se encuentran las contribuciones de De Gregorio $(1992,2007)$ y De Gregorio y Lee (1999), así como Bittencourt (2012), entre otros. Por último, una cuarta categoría de trabajos destacan el rol negativo que han tenido las estrategias de desarrollo hacia adentro y sin reformas económicas que tiendan a removerlas como De Gregorio y Lee (1999), Hopenhayn y Neumeyer (2004), y Rodrigues (2010). En este sentido, Dabús, González y Bermúdez (2012, p. 4) indican que En particular, esta línea de trabajos encuentra que la 
aplicación de reformas insuficientes y un fuerte sesgo mercado internista darían cuenta de buena parte del bajo crecimiento de América Latina en la segunda mitad del siglo pasado.

Por otro lado, diversos autores han analizado el proceso de crecimiento en Latinoamérica con base en la vinculación del mismo con la inestabilidad macroeconómica, la que según la evidencia presentada en la literatura tuvo un rol central en dicho proceso. Entre los estudios sobre el tema se encuentran los de Loayza, Fajnzylber y Calderón (2003) y Sahay y Goyal (2007). En particular, el trabajo de Loayza, Fajnzylber y Calderón (2003) diferencia la tendencia del componente cíclico del producto. Este análisis permite destacar que los períodos de mayor volatilidad, dada por la amplitud de los ciclos, se asocian a una menor tendencia de crecimiento.

En ese sentido, Catao (2007) profundiza el análisis sobre la conducta de las fluctuaciones económicas en América Latina a partir de la reconstrucción de datos sobre el producto desde 1870, de modo de poder obtener un enfoque de largo plazo sobre la volatilidad en la región. El autor encuentra, en primer lugar, que la volatilidad ha sido mayor en América Latina que en el resto del mundo. Esta tendencia se ha repetido a lo largo de todo el período analizado (1870-2004), y es notoria en la etapa previa a la crisis de 1930, cuando las fluctuaciones del producto de los países líderes (Argentina, Brasil, Méjico y Chile) fueron mayores que en países similares del resto del mundo. En segundo lugar, destaca la existencia de una conducta similar en la evolución de los ciclos en los países de la región, lo que sugiere la existencia de un fuerte componente externo en común que afecta dicha conducta. Según su estudio, las principales variables que actuaron como shocks externos coordinadores de los ciclos fueron las variaciones del producto y la tasa de interés real de las economías avanzadas.

A su vez, Fanelli y Jiménez (2010) identifican una serie de hechos estilizados asociados a la volatilidad macroeconómica; destacan que la misma resulta una característica intrínseca de Latinoamérica, y que ha sido mayor aquí que en el resto del mundo. Al mismo tiempo, encuentran que dicha volatilidad tiene un efecto perjudicial sobre el desempeño de la economía. Por lo tanto, en forma compatible a los trabajos antes mencionados, los autores concluyen que la reducción de la volatilidad es un objetivo prioritario para promover el crecimiento económico. En el mismo sentido, en un estudio para América Latina para el período 1950-2009, Bermúdez, Dabús y González (2015) encuentran que la inestabilidad, aproximada por la inflación y la volatilidad de la tasa de crecimiento del producto perjudican al crecimiento en Latinoamérica.

En síntesis, aunque los determinantes de los procesos de crecimiento y estancamiento en la región han sido múltiples, parece haber un consenso en la literatura 
respecto a que la inestabilidad ha sido perjudicial para el crecimiento, lo que será analizado más adelante en la sección 3.

\section{DATOS Y METODOLOGÍA}

Los datos de las variables en estudio corresponden al período 1980-2014, el que fue elegido de acuerdo con la disponibilidad de datos. A su vez, se toma un conjunto de 17 países de Latinoamérica; estos son Argentina, Bolivia, Brasil, Chile, Colombia, Costa Rica, República Dominicana, Ecuador, El Salvador, Guatemala, Honduras, México, Nicaragua, Panamá, Paraguay, Perú, Uruguay y Venezuela.

Los datos son tomados en promedio quinquenal para cada variable. Estos han sido extraídos de dos fuentes: el Banco Mundial y la CEPAL. En particular, todas las variables son tomadas del Banco Mundial, excepto la tasa de inflación, la que corresponde a la base de datos de la CEPAL, debido a que esta base contiene datos más completos sobre dicha variable. La tabla 1 muestra una descripción completa de cada una de las variables utilizadas².

\section{Tabla 1. Descripción de las variables}

\begin{tabular}{|l|l|l|}
\hline \multicolumn{1}{|c|}{ Variables } & \multicolumn{1}{c|}{ Descripción } & \multicolumn{1}{c|}{ Fuente } \\
\hline Crecimiento & Crecimiento del PBI (\%) & Banco Mundial \\
\hline Gini & Indice de Gini & $\begin{array}{l}\text { Estimaciones del Banco } \\
\text { Mundial }\end{array}$ \\
\hline Inversión/PBI & Formación bruta de capital (\%) & Banco Mundial \\
\hline Capital Humano & Matricula secundario, ambos sexos (\%) & Banco Mundial \\
\hline Apertura & $\begin{array}{l}\text { Intercambio (exportaciones más } \\
\text { importaciones), como \%PBI }\end{array}$ & Banco Mundial \\
\hline Crecimiento Poblacional & \% anual & Banco Mundial \\
\hline Medidas de volatilidad & \multicolumn{2}{|l|}{} \\
\hline Inflación & Inflación precios del consumidor (\%) & CEPAL \\
\hline SD & $\begin{array}{l}\text { Desvió estándar quinquenal del } \\
\text { crecimiento }\end{array}$ & $\begin{array}{l}\text { Elaboración propia con base } \\
\text { en datos del Banco Mundial }\end{array}$ \\
\hline
\end{tabular}

Fuente: elaboración propia.

En relación con la metodología empleada se trabaja con regresiones de panel, y se considera que existen efectos intrínsecos a cada país, de acuerdo con lo indicado en la ecuación (1), como sigue:

2 La base de datos usada en este estudio puede ser solicitada por cualquier lector al correo: cdabus@ criba.edu.ar. 


$$
y_{i t}=c_{i}+\beta x_{i, t}+\varepsilon_{i t}
$$

donde $c_{i}$ es el efecto individual, también llamado heterogeneidad no observada, y $x_{i, t}$ contiene el conjunto de variables explicativas antes mencionadas. En efecto, como se mencionó, en este caso las variables utilizadas son la tasa de crecimiento del producto como variable endógena, y entre las explicativas se encuentran las de control utilizadas en la literatura, de acuerdo con los resultados encontrados en el trabajo de Levine y Renelt (1992), es decir, el cociente Inversión/PBI, el capital humano, el coeficiente de apertura (medido como la suma de exportaciones sobre importaciones en relación al producto), y la tasa de crecimiento poblacional. A su vez, en función de la evidencia presentada en la literatura de crecimiento antes citada, a estas se agregan como variables explicativas el coeficiente de Gini como indicador de desigualdad y las variables de inestabilidad antes mencionadas, es decir, la volatilidad de la tasa de crecimiento del producto y la inflación.

Por otro lado, en las estimaciones el efecto individual puede ser fijo o aleatorio, según esté o no correlacionado con las variables explicativas. Si no se encuentra correlacionado, puede ser tratado como un residuo. Si por otro lado se encuentra que la covarianza entre las variables contenidas en $x$ y el efecto individual es distinta de cero, la ecuación debe ser transformada de manera de poder estimar los coeficientes sin sesgo. En este caso, el test $F$ indica que no se rechaza (con un p-valor de 0,3788) la hipótesis nula de que todos los efectos fijos son iguales a cero, por lo que las regresiones se especifican con efectos aleatorios. Esto resultado se puede deber a que la región es relativamente homogénea en cuanto a las variables explicativas, en particular, las relacionadas con la inestabilidad económica.

No se utiliza la técnica de Arellano y Bond (1991) o Blundell y Bond (1998) para hacer paneles dinámicos, dado que no solo las variables resultaron no significativas, sino que, además, los tests de Sargan de validez de instrumentos no validaron los mismos. Esto se puede deber a que las observaciones son expresadas en promedio quinquenal, en particular las correspondientes a las variables de inestabilidad (es decir, la volatilidad de la tasa de crecimiento producto y la inflación). Por tanto, el estudio se realizó solo con base en los efectos contemporáneos entre las variables.

Por otra parte, se realizó una clasificación de la muestra en diferentes regímenes de crecimiento a partir del método de clúster de medianas, el que arrojó la existencia de tres clúster: uno de crecimiento alto, uno de medio y uno de bajo, con valores promedio de crecimiento del producto per cápita para cada clúster de 5,1 \%, 2,4 \% y $-1,3 \%$, respectivamente. La elección del método de clúster de medianas obedece a que este logra armar grupos de observaciones que se ven menos influidas por valores atípicos. De hecho, se eligió este método porque las variables de crecimiento 
e inflación para América Latina han tenido períodos con valores extremos, es decir, muy altos o muy bajos, como se verá en la tabla 1 de la sección siguiente.

Finalmente, se incluyeron en el estudio empírico variables que interactúan entre el régimen de crecimiento y las variables de inestabilidad. La inclusión de estas variables permite captar aspectos interesantes a partir de la interpretación que puede darse a las mismas. Cuando una variable indicadora o dummy (en este caso el régimen de crecimiento) interactúa con una variable continua (aquí la volatilidad de la tasa de crecimiento del producto o la inflación), el resultado de la estimación indica el efecto de esta última en el grupo al que hace referencia la variable indicadora.

\section{EVIDENCIA EMPÍRICA}

En esta sección se presenta la evidencia empírica sobre la relación entre la inestabilidad económica y el crecimiento en América Latina. La siguiente tabla presenta la estadística descriptiva de las variables en estudio, en particular los valores promedio y la desviación estándar de las mismas.

Tabla 2. Estadística descriptiva, 1980-2014 (promedios de valores quinquenales)

\begin{tabular}{|l|c|c|c|c|c|}
\hline \multicolumn{1}{|c|}{ Variable } & Obs & Promedio & $\begin{array}{c}\text { Desvío } \\
\text { Standard }\end{array}$ & $\begin{array}{c}\text { Valor } \\
\text { Mínimo }\end{array}$ & $\begin{array}{c}\text { Valor } \\
\text { Máximo }\end{array}$ \\
\hline Crecimiento & 126 & 3,2 & 2,3 & $-5,1$ & 8,3 \\
\hline Gini & 105 & 51,5 & 6,9 & 40,2 & 99,9 \\
\hline Inversión/PBI & 124 & 20,3 & 4,0 & 12,6 & 32,1 \\
\hline Capital Humano & 114 & 63,6 & 19,2 & 18,7 & 104,4 \\
\hline Inflación (CEPAL) & 124 & 119,8 & 428,1 & 0,5 & 2728,5 \\
\hline Apertura & 126 & 44,8 & 21,5 & 12,2 & 112,0 \\
\hline Crecimiento Poblacional & 126 & 1,7 & 0,6 & 0,1 & 3,1 \\
\hline SD & 126 & 3,2 & 2,1 & 0,3 & 11,0 \\
\hline
\end{tabular}

Fuente: elaboración propia con datos del Banco Mundial y CEPAL para el caso de la inflación

La tabla 1 muestra niveles promedio de crecimiento y del cociente inversión/ PBI relativamente bajos para el período en estudio. A su vez, se observa una gran dispersión de las variables consideradas, en particular de la tasa de crecimiento, el coeficiente de Gini, el coeficiente de apertura y la tasa de inflación. Esto indicaría que la región ha tenido gran inestabilidad en el período de tiempo en estudio. Asimismo, el hecho de que se verifique una marcada dispersión en la tasa de crecimiento del producto sugiere una evolución muy fluctuante del mismo. Del mismo 
modo, los valores muy dispares de la tasa de inflación indican que en el período en estudio la región ha experimentado desde episodios de baja inflación hasta casos de severas hiperinflaciones, como los sufridos por países como Argentina, Bolivia, Perú y Nicaragua durante la década del 1980, entre otros.

En tal sentido, como indica la tabla 1 la inflación tuvo una evolución que va desde valores anuales (en promedio quinquenal) cercanos a cero, a máximos de $2.728,5 \%$ para toda la muestra. A su vez, se observa que el producto también ha tenido una alta volatilidad en su evolución, con períodos de gran crecimiento (los que han superado el $8 \%$ anual promedio en un quinquenio) y otros de fuertes caídas del producto, los que llegaron a convertirse en recesiones con tasas de crecimiento negativas del orden del $5 \%$ anual en promedio.

Por otra parte, como se vio en la introducción, la literatura existente en general muestra una relación negativa entre inestabilidad y crecimiento económico. Dada la historia de inestabilidad en Latinoamérica, resulta relevante considerar la evidencia preliminar de dicha relación a partir de un análisis gráfico, el cual se incluye en el anexo. Los gráficos 1, 2, 3 y 4 muestran la relación entre la inestabilidad, aproximada por la desviación estándar del crecimiento, con el crecimiento para la muestra global y para los países que pertenecen a cada uno de los regímenes en particular.

El método no paramétrico utilizado para el ajuste en los gráficos 1 a 4 es el de suavizado de Kernel o kernel smoothing como lo plantea Fan (1992). En el mismo, se parte de una nube de puntos $\left\{\left(\mathrm{x}_{1}, \mathrm{y}_{1}\right), \ldots,\left(\mathrm{x}_{\mathrm{n}}, \mathrm{y}_{\mathrm{n}}\right)\right\}$ y un modelo del estilo $m\left(x_{i}\right)+\sigma\left(x_{i}\right) \varepsilon_{i}$ donde $m\left(\right.$.) denota la media y $\sigma^{2}$ (.) la varianza, ambas desconocidas, con errores $\epsilon_{\mathrm{i}}$ donde $\mathrm{E}\left(\epsilon_{\mathrm{I}}\right)=0$ y $\operatorname{Var}\left(\epsilon_{\mathrm{I}}\right)=1$. La estimación es hecha entonces en función de $\mathrm{m}\left(\mathrm{x}_{0}\right)=\mathrm{E}\left[\mathrm{Y} \mid \mathrm{X}=x_{0}\right]$. De esa manera, el ajuste es local al entorno de $x_{0}$. No se hace ningún supuesto en la forma funcional de $m($.$) . En este trabajo, el kernel es$ especificado como una distribución Epanechnikov (aunque los resultados no varían si se elige una distribución gaussiana).

El análisis gráfico realizado por este método permite observar que se verifica una relación negativa entre la inestabilidad y el crecimiento para el total de la muestra (ver gráfico 1), aunque para los países de crecimiento alto esta relación no es tan clara (ver gráfico 2). A su vez, es más claro el efecto en el caso de los países con régimen de ingreso medio y bajo, lo cual puede observarse en los gráficos 3 y 4 . Por tanto, el análisis gráfico muestra que, a excepción del caso de los países de crecimiento alto, existe una relación negativa entre inestabilidad y crecimiento, lo que, a continuación, se analiza de forma econométrica a fin de tener una aproximación más precisa sobre dicha relación. 
Gráfico 1. Relación entre Inestabilidad (SD) y Crecimiento del PBI per cápita en América Latina (GDPPC): 1980-2014, total de la muestra de países

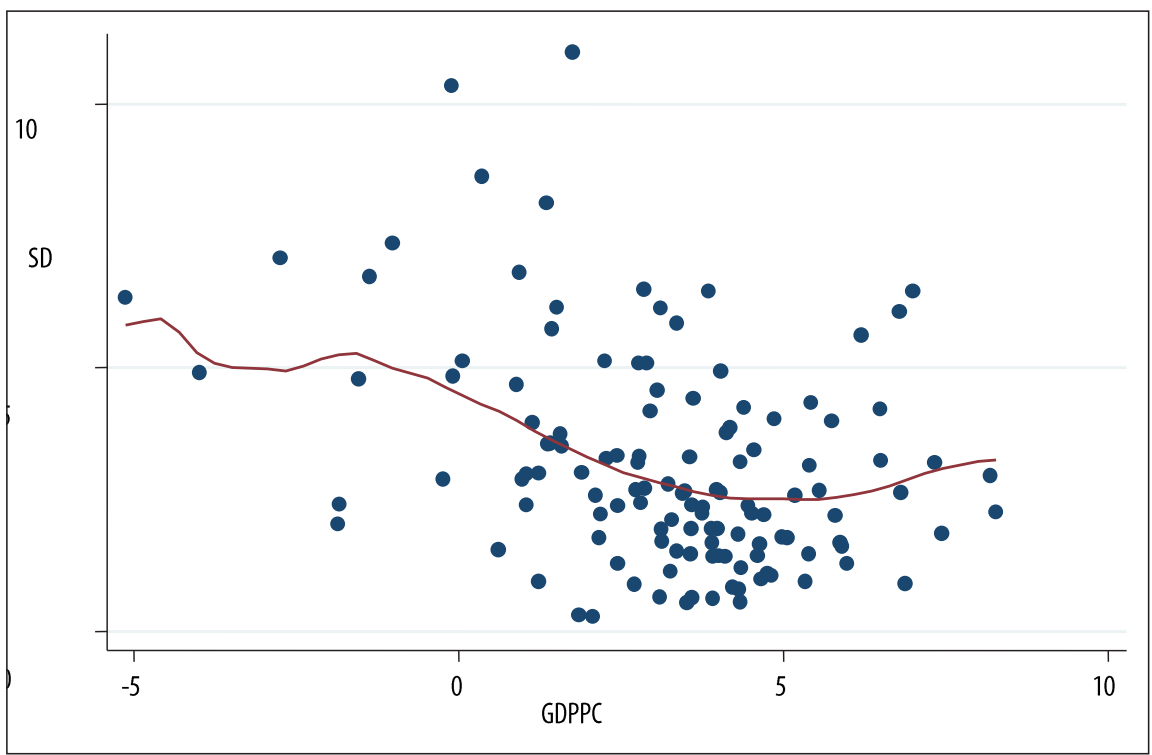

Fuente: elaboración propia con base en datos del Banco Mundial y la CEPAL

Gráfico 2. Relación entre Inestabilidad (SD) y Crecimiento del PBI per cápita (GDPPC) en América Latina: 1980-2014, países con régimen de crecimiento alto

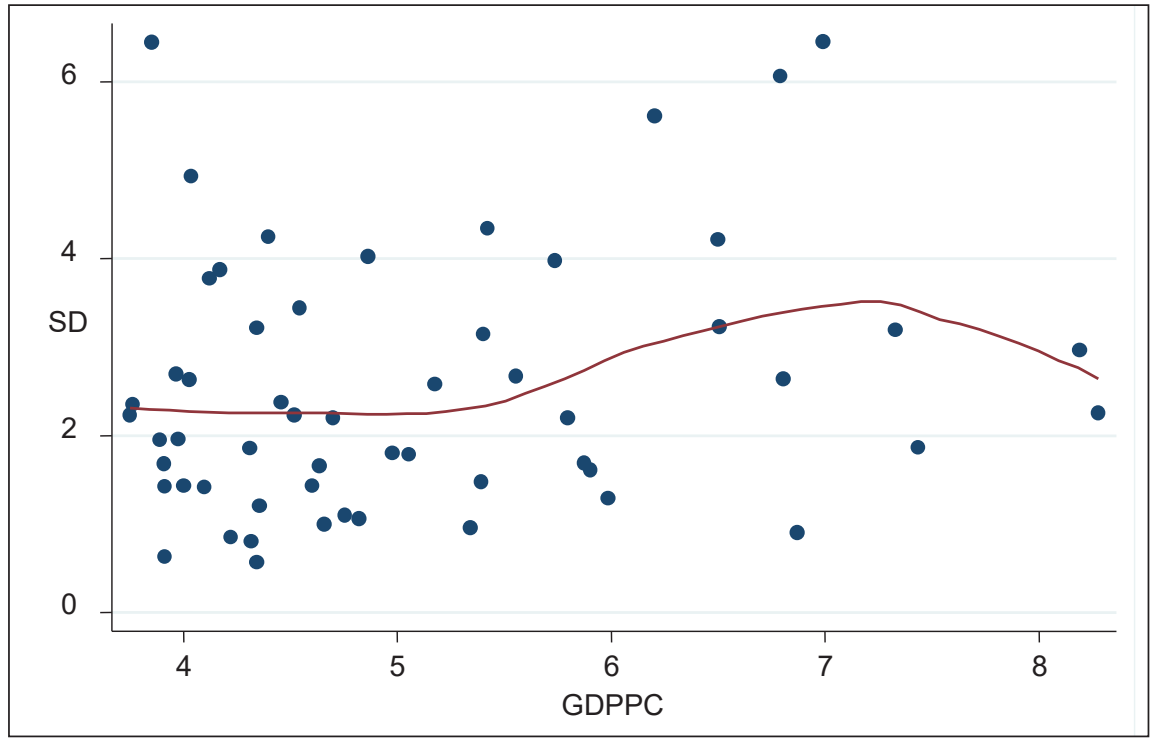

Fuente: elaboración propia con base en datos del Banco Mundial y la CEPAL 


\subsection{RESULTADOS DE LAS REGRESIONES}

La tabla 2 muestra los resultados de las estimaciones realizadas por medio de datos de panel. La primera columna estima el modelo de efectos aleatorios con las variables de control y de interés, pero sin incluir las variables de interacción. La segunda columna muestra el modelo de efectos fijos que, como se indicó, se descartó como consecuencia de los resultados de los test realizados, aunque se muestra de manera de corroborar que los resultados no varían de forma sustancial al incluirlos. A su vez, las columnas 3, 4 y 5 presentan los resultados de las estimaciones cuando se utilizan las variables de interacción. Se emplea como proxy de la inestabilidad de la desviación estándar de la tasa de crecimiento del producto, mientras que las columnas 6, 7 y 8 muestran el mismo análisis, pero al incluir como proxy la tasa de inflación.

Tabla 3. Inestabilidad y crecimiento en América Latina, 1980-2014

\begin{tabular}{|c|c|c|c|c|c|c|c|c|}
\hline & EA & $\mathrm{EF}$ & EA & EA & EA & EA & EA & EA \\
\hline Variables & (1) & (2) & (3) & (4) & (5) & (6) & (7) & (8) \\
\hline \multirow[t]{2}{*}{ Inversión/PBI } & $0,111^{*}$ & $0,176^{* *}$ & $0,0779 *$ & $0,123^{* *}$ & 0,061 & $0,109 *$ & $0,111^{*}$ & $0,107^{*}$ \\
\hline & $(0,061)$ & $(0,081)$ & $(0,044)$ & $(0,060)$ & $(0,055)$ & $(0,060)$ & $(0,061)$ & $(0,060)$ \\
\hline \multirow[t]{2}{*}{ KHum } & $0,029^{*}$ & $-0,008$ & 0,006 & 0,024 & 0,0255 & $0,031^{*}$ & $0,030^{*}$ & $0,030^{*}$ \\
\hline & $(0,017)$ & $(0,035)$ & $(0,013)$ & $(0,017)$ & $(0,016)$ & $(0,017)$ & $(0,017)$ & $(0,017)$ \\
\hline \multirow[t]{2}{*}{ Openess } & 0,0139 & 0,0215 & 0,006 & 0,013 & 0,0104 & 0,0153 & 0,0150 & 0,0119 \\
\hline & $(0,011)$ & $(0,018)$ & $(0,008)$ & $(0,011)$ & $(0,010)$ & $(0,011)$ & $(0,011)$ & $(0,011)$ \\
\hline \multirow[t]{2}{*}{ GPop } & 0,344 & $-0,166$ & 0,164 & 0,372 & 0,109 & 0,329 & 0,334 & 0,355 \\
\hline & $(0,520)$ & $-1,301$ & $(0,416)$ & $(0,510)$ & $(0,493)$ & $(0,513)$ & $(0,520)$ & $(0,511)$ \\
\hline \multirow[t]{2}{*}{ GINI } & $-0,028$ & $-0,063$ & $-0,006$ & $-0,039$ & 0,026 & $-0,023$ & $-0,024$ & $-0,034$ \\
\hline & $(0,031)$ & $(0,039)$ & $(0,022)$ & $(0,030)$ & $(0,029)$ & $(0,030)$ & $(0,031)$ & $(0,030)$ \\
\hline \multirow[t]{2}{*}{ Inf } & $-0,0002$ & $-0,0002$ & $-0,0003$ & $-0,0003$ & 0,00002 & $-0,0010$ & 0,00020 & $-0,00009$ \\
\hline & $(0,0005)$ & $(0,0006)$ & $(0,0003)$ & $(0,0005)$ & $(0,0004)$ & $(0,0006)$ & $(0,0007)$ & $(0,0005)$ \\
\hline \multirow[t]{2}{*}{ SD } & $-0,325 * * *$ & $-0,353 * *$ & $-0,387 * * *$ & $-0,222 * *$ & $-0,00988$ & $-0,300 * * *$ & $-0,320 * * *$ & $-0,283^{* * *}$ \\
\hline & $(0,101)$ & $(0,142)$ & $(0,073)$ & $(0,110)$ & $(0,107)$ & $(0,101)$ & $(0,101)$ & $(0,101)$ \\
\hline \multirow[t]{2}{*}{ SD-alto } & & & $0,880^{* * *}$ & & & & & \\
\hline & & & $(0,087)$ & & & & & \\
\hline SD-medio & & & & $-0,232 * *$ & & & & \\
\hline
\end{tabular}


Inestabilidad, crecimiento y desempeño económico: evidencia de 17 países de América Latina, 1980-2014

\begin{tabular}{|c|c|c|c|c|c|c|c|c|}
\hline & EA & $\mathrm{EF}$ & EA & EA & EA & EA & EA & EA \\
\hline \multirow[t]{2}{*}{ Variables } & (1) & (2) & (3) & (4) & (5) & (6) & (7) & (8) \\
\hline & & & & $(0,109)$ & & & & \\
\hline \multirow[t]{2}{*}{ SD-bajo } & & & & & $-0,673 * * *$ & & & \\
\hline & & & & & $(0,120)$ & & & \\
\hline \multirow[t]{2}{*}{ INF-alto } & & & & & & $0,001^{*}$ & & \\
\hline & & & & & & $(0,001)$ & & \\
\hline \multirow[t]{2}{*}{ INF-medio } & & & & & & & $-0,0009$ & \\
\hline & & & & & & & $(0,001)$ & \\
\hline \multirow[t]{2}{*}{ INF-bajo } & & & & & & & & $-0,004^{* *}$ \\
\hline & & & & & & & & $(0,002)$ \\
\hline \multirow[t]{2}{*}{ Constante } & 0,659 & 4,181 & 1,396 & 1,307 & $-1,022$ & 0,196 & 0,355 & 0,964 \\
\hline & $(-2,221)$ & $(-4,669)$ & $(-1,702)$ & $(-2,199)$ & $(-2,084)$ & $(-2,209)$ & $(-2,247)$ & $(-2,190)$ \\
\hline $\begin{array}{l}\text { Observacio- } \\
\text { nes }\end{array}$ & 95 & 95 & 95 & 95 & 95 & 95 & 95 & 95 \\
\hline R2 & 0,249 & 0,200 & 0,640 & 0,287 & 0,432 & 0,276 & 0,257 & 0,282 \\
\hline
\end{tabular}

Nota: EA indica efectos aleatorios y EF efectos fijos. Los errores estándar están entre paréntesis, y la significatividad es como sigue: ${ }^{* * *}$ es significativa al $1 \%,{ }^{* *}$ al $5 \%$ y $*$ al $10 \%$. A su vez, alto medio y bajo indican régimen de crecimiento alto, medio y bajo, respectivamente.

Fuente: elaboración propia con base en datos del Banco Mundial y la CEPAL

En relación con las variables de control, en la tabla 2 se observa que en general la variable inversión/PBI es significativa y con el signo esperado, resultado que a largo plazo, como bien se explica en el trabajo de Gutiérrez, Rendón y Álvarez (2004), no se obtiene del tradicional modelo de Solow-Swan. De hecho, según este modelo, el ahorro y la inversión solo tienen efectos transitorios sobre la tasa de crecimiento. Con respecto al resto de las variables en estudio el capital humano solo en algunos casos afecta positiva y significativamente al crecimiento. A su vez, en forma compatible con la evidencia encontrada en el trabajo de Delbianco, Dabús y Caraballo (2014), la desigualdad en general afecta negativamente al crecimiento.

Por otro lado, la inestabilidad aproximada por la tasa de inflación no resulta significativa. Por el contrario, si se mide como el desvío estándar de la tasa de crecimiento del producto resulta significativa y con el signo esperado (negativo), por lo que los resultados indican que en particular la volatilidad del producto sería 
relevante en términos del impacto negativo de la inestabilidad sobre el crecimiento a largo plazo en la región. Este resultado es compatible con parte de la literatura antes citada, como, por ejemplo, Sahay y Goyal (2007) y Fanelli y Jiménez (2010).

En relación con los resultados por clúster o regímenes de crecimiento, de la interacción entre estos y la inestabilidad se observa que existe una clara diferencia entre el régimen de crecimiento alto con relación al resto. De forma contraria a lo esperado, cuando las economías de la región experimentan períodos de crecimiento alto ambas medidas de inestabilidad lo afectan de forma positiva y significativa. Sin embargo, en los regímenes de crecimiento medio y bajo en general ambas variables de inestabilidad son significativas y con el signo esperado, es decir, afectan negativamente al crecimiento. En particular, la volatilidad del producto impacta negativa y significativamente sobre el crecimiento cuando el régimen es medio o bajo.

Por tanto, en estos casos parece clave evitar fuertes fluctuaciones del producto por medio de políticas anticíclicas para generar un entorno más favorable para el crecimiento. Finalmente, cuando el crecimiento es bajo tanto la volatilidad del producto como la inflación lo afectan negativamente. De este modo, el resultado más interesante parece darse en los regímenes de menor crecimiento. En tal sentido, en estos casos no solo sería recomendable la aplicación de políticas anticíclicas, sino también planes de estabilización de precios que eviten episodios de alta inflación.

\section{CONCLUSIONES}

En este trabajo se analizó la relación entre inestabilidad económica y crecimiento para Latinoamérica durante el período 1980-2014; el estudio se enfocó en diferentes regímenes de crecimiento: alto, medio y bajo. Estos regímenes fueron obtenidos por medio del método de clúster de medianas, a fin de evitar la influencia de los valores extremos en la obtención de los regímenes.

La inestabilidad es aproximada por la volatilidad de la tasa de crecimiento del producto y la inflación. La evidencia encontrada indica que en períodos de alto crecimiento la inestabilidad no afecta negativamente al crecimiento, por lo que en estos casos la región parece ser impulsada por factores ajenos a la inestabilidad en sí. En particular, dado que la misma está constituida por un conjunto de países con un mercado interno pequeño, en los períodos de gran crecimiento podría ser relevante un contexto externo favorable que impulse de forma exógena a los países de la región.

Por el contrario, en regímenes de crecimiento medio o bajo, sí son perjudiciales para el desempeño económico las variables asociadas a la inestabilidad, y en 
particular la volatilidad del crecimiento del producto. Este resultado sugiere que en estos regímenes deberían aplicarse políticas destinadas a minimizar la inestabilidad. En particular, parece clave la aplicación tanto de políticas anticíclicas, de modo de reducir la volatilidad del producto y por tanto mantener un sendero de crecimiento relativamente estable, así como de políticas antiinflacionarias que permitan evitar episodios de desbordes inflacionarios, como los que ha experimentado la región en la década de 1980.

Como posibles extensiones a este trabajo pueden incorporarse medidas alternativas de inestabilidad, tales como la volatilidad de la tasa de inflación y de variables macroeconómicas clave como el tipo de cambio. Finalmente, otra alternativa sería ampliar la muestra de países a otras regiones de modo de poder comparar el impacto de la inestabilidad sobre el crecimiento entre Latinoamérica y otras regiones más estables.

\section{BIBLIOGRAFÍA}

Astorga, Pablo (2010). A century of economic growth in Latin America. En: Journal of Development Economics, Vol. 92, No. 2, p. 232-243.

Arellano, Manuel y Bond, Stephen (1991). Some tests of specification for panel data: Monte Carlo evidence and an application to employment equations. En: The Review of Economic Studies, Vol. 58, No. 2, p. 277-297.

Bermúdez, Cecilia, Dabús, Carlos y González, Germán (2015). Reexamining the link between instability and growth in Latin America: a dynamic panel data estimation using K-Median clusters. En: Latin America Journal of Economics, Vol. 52, No. 1, p. 1-23.

Bittencourt, Manoel (2012). Inflation and economic growth in Latin America: some panel time-series evidence. En: Economic Modelling, Vol. 29, No. 2, p. 333-340.

Blundell, Richard y Bond, Stephen (1998). Initial conditions and moment restrictions in dynamic panel data models. En: Journal of Econometrics, Vol. 87, No. 1, p. 115 143.

Brizeño, Rodrigo, Cercone, James y Cardoza, David (2003). ¿Es la inversión en capital h umano la vía para el fomento del crecimiento económico? El caso de Costa Rica. En: Instituto Latinoamericano de Políticas Públicas (ILAPP), 44p.

Catao, Luis (2007). Retrospectiva latinoamericana. En: Finanzas y Desarrollo, Vol. 44, No. 4, p. $39-43$.

Chumachero, Rómulo y Fuentes, Rodrigo (2006). Economic Growth in Latin America: Structural Breaks or Fundamentals? En: Estudios de Economía, Vol. 33, No. 002, p. 141-154.

Cimoli, Mario; Pereira, Wellington; Porcile, Gabriel y Dória Scatolin, Fábio (2011). Structural change, technology, and economic growth: Brazil and the CIBS in a comparative perspective. En: Economic Change and Restructuring, Vol. 44, No. 1, p. 25-47. 
Clavijo, Sergio (2003). Growth and productivity in Colombia. En: Documento de trabajo, Banco de la República, Colombia, p. 1-21.

Coremberg, Ariel (2006). Fuentes del Crecimiento Económico en Argentina 1990-2004. ¿Otro Caso de la Tiranía de los Números? En: Revista de Economía Política de Buenos Aires, Vol. 1, No. 2, p. 55 88.

Dabús, Carlos, González, Germán y Bermúdez, Cecilia (2012). Inestabilidad y crecimiento económico: evidencia de América Latina, p. 117-174. En: Keifman, Saúl (editor), Progresos en Crecimiento Económico, Ciudad Autónoma de Buenos Aires, Fondo Editorial Consejo, 323 p.

De Gregorio, José (1992). Economic growth in Latin America. En: Journal of Development Economics, Vol. 39, No. 1, p. 59-84.

De Gregorio, J. (2007). Algunas reflexiones sobre el crecimiento económico en Chile. En: Economic Policy Papers, Banco Central de Chile, No. 20, p. 1-14.

De Gregorio, José y Lee, Jong-Wha (1999). Economic growth in Latin America: sources and prospects. En: Documentos de Trabajo, Centro de Economía Aplicada, Universidad de Chile, No. $9 \overline{6,39 p .}$

Deininger, Klaus y Squire, Lyn (1996). A new data set measuring income inequality. En: The World Bank Economic Review, Vol. 10, No. 3, p. 565-591.

Delbianco, Fernando; Dabús, Carlos y Caraballo Pou, María Ángeles (2014). Income inequality and economic growth: new evidence from Latin America. En: Cuadernos de Economía, Vol. 33, No. 63, p. 381-398.

Durán Ortiz, Juan Pablo y Hurtado Rendón, Álvaro Arturo (2009). Una explicación alternativa del crecimiento económico colombiano a la relación crecimiento-seguridad democrática. En: Economía Autónoma, No. 2, p. 24-40.

Fan, Jianqing (1992). Design-adaptive nonparametric regression. En: Journal of the American Statistical Association, Vol. 87, No. 420, p. 998-1004.

Fanelli, José Maŕa y Jiménez, Juan Pablo (2010). Volatilidad macroeconómica y espacio fiscal en América Latina, p. 157-181. En: Alonso, José Antonio y Bárcena, Alicia (coordinadores): Retos y oportunidades ante la crisis, Madrid, Pensamiento Iberoamericano, No 6.

Gutiérrez Londoño, Eber Elí (1999). Crecimiento económico en América Latina. En: Semestre Económico, Vol. 2, No. 4, p. 1-4.

Gutiérrez Londoño, Eber Elí; Rendón Acevedo, Jaime Alberto y Álvarez García, Rubén Darío (2004). El crecimiento económico en el modelo de Solow y aplicaciones. En: Semestre Económico, Vol. 7, No. 14, p. 16-29.

Hopenhayn, Hugo y Neumeyer, Pablo Andrés (2004). Latin America in the XXth century: stagnation, then collapse. En: Econometric Society 2004, Latin American Meetings, No. 326, 28p.

Kuznets, Simon (1955). Economic growth and income inequality. En: American Economic Review, Vol. 45, No. 1, 28p. 
Levine, Ross Eric y Renelt, David (1992). A sensitivity analysis of cross-country regressions. En: American Economic Review, Vol. 82, No. 4, p. 942-963.

Loayza, Norman; Fajnzylber, Pablo y Calderón, César (2003). Economic growth in Latin America and the Caribbean. Stylized facts, explanations and forecasts. En: Banco Central de Chile, Documentos de trabajo No. 265,150 p.

Manuelli, Rodolfo (2005). Growth in Latin America: empirical findings and some simple theoretical explanations, p. 55-116. En: Fernández-Arias, Eduardo; Manuelli, Rodolfo y Blyde, Juan (Eds.). Sources of growth in Latin America. What is missing? Washington D.C., Editorial Inter-American Development Bank, 478p.

Pardo, Jimena y Reig, Nicolás (2002). Crecimiento, demanda y exportaciones en la economía uruguaya: 1960-2000. En:, Facultad de Ciencias Sociales, Universidad de la República, Documento de Trabajo No. 11, 62p.

Rodrigues, Mauro (2010). Import substitution and economic growth. En: Journal of Monetary Economics, Vol. 57, No. 2, p. 175-188.

Sahay, Ratna y Goyal, Rishi (2007). Volatility and growth in Latin America: an episodic a pproach. En: International Monetary Fund Working Paper, No. 06/287, 54p.

Singh, Anoop y Cerisola, Martín (2006). Sustaining Latin America's resurgence: some historical perspectives. En: International Monetary Fund Working Paper, No. 06/252, 39p. 


\section{ANEXO}

Gráfico 3. Relación entre Inestabilidad (SD) y Crecimiento del PBI per cápita (GDPPC) en América Latina: 1980-2014, países con régimen de crecimiento medio

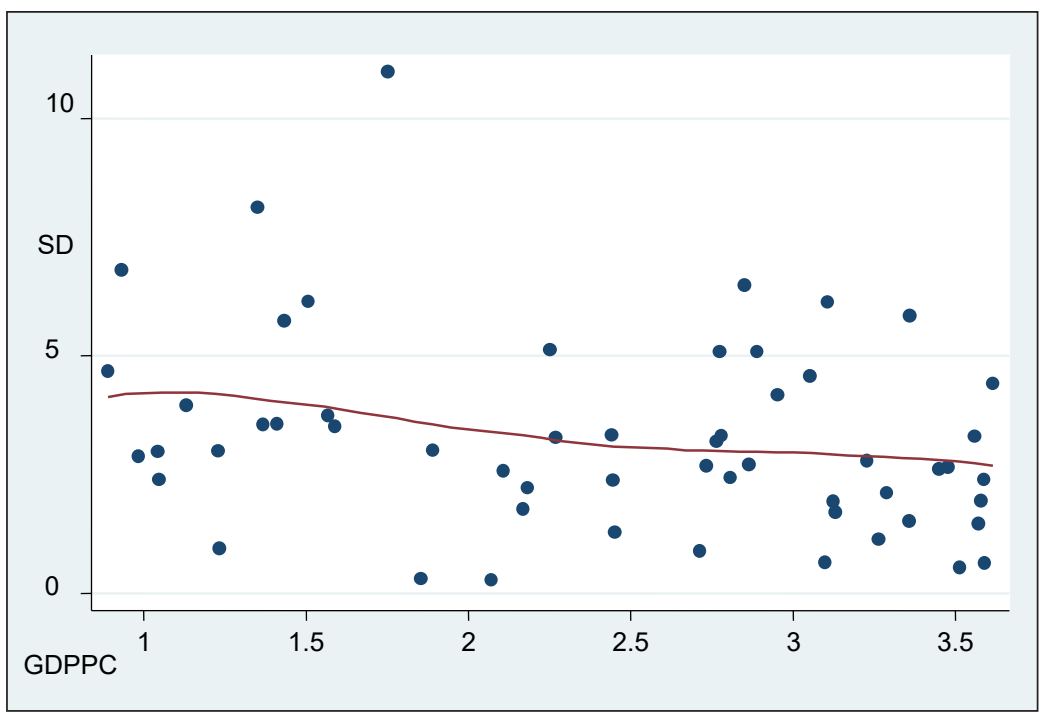

Fuente: elaboración propia con base en datos del Banco Mundial y la CEPAL

Gráfico 4. Relación entre Inestabilidad (SD) y Crecimiento del PBI per cápita (GDPPC) en América Latina: 1980-2014, países con régimen de crecimiento bajo

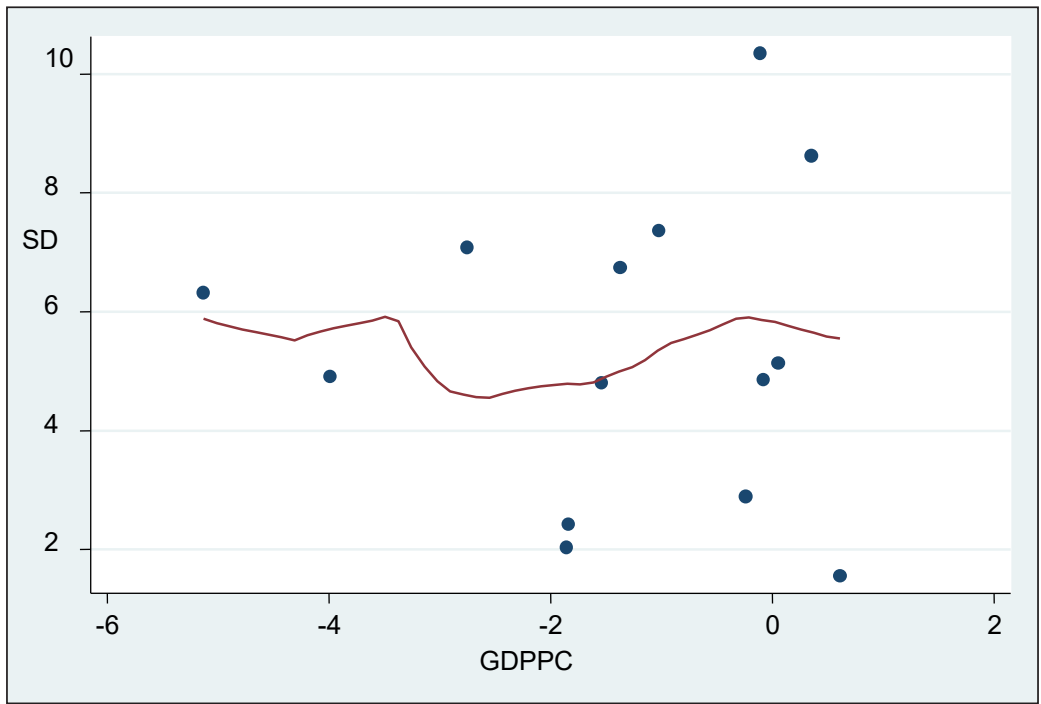

Fuente: elaboración propia con base en datos del Banco Mundial y la CEPAL 
Inestabilidad, crecimiento y desempeño económico: evidencia de 17 países de América Latina, 1980-2014

Tabla 4. Régimen de crecimiento por país y quinquenio

\begin{tabular}{|c|c|c|c|c|c|}
\hline \multicolumn{2}{|c|}{ Alto } & \multicolumn{2}{|c|}{ Medio } & \multicolumn{2}{|c|}{ Bajo } \\
\hline País & Quinquenio & País & Quinquenio & País & Quinquenio \\
\hline Argentina & 1990-1994 & Argentina & 1995-1999 & Argentina & $1980-1984$ \\
\hline Argentina & $2005-2009$ & Bolivia & 1985-1989 & Argentina & 1985-1989 \\
\hline Argentina & 2010-2014 & Bolivia & 2000-2004 & Argentina & 2000-2004 \\
\hline Bolivia & 1990-1994 & Brasil & 1980-1984 & Bolivia & 1980-1984 \\
\hline Bolivia & 1995-1999 & Brasil & 1990-1994 & Costa Rica & 1980-1984 \\
\hline Bolivia & $2005-2009$ & Brasil & 1995-1999 & El Salvador & 1980-1984 \\
\hline Bolivia & 2010-2014 & Brasil & 2000-2004 & Guatemala & 1980-1984 \\
\hline Brasil & 1985-1989 & Brasil & 2005-2009 & Nicaragua & $1985-1989$ \\
\hline Chile & 1985-1989 & Brasil & 2010-2014 & Nicaragua & 1990-1994 \\
\hline Chile & 1990-1994 & Chile & 1980-1984 & Panamá & $1985-1989$ \\
\hline Chile & 1995-1999 & Chile & 2005-2009 & Perú & $1985-1989$ \\
\hline Chile & 2000-2004 & Colombia & 1980-1984 & Uruguay & 1980-1984 \\
\hline Chile & 2010-2014 & Colombia & 1995-1999 & Uruguay & 2000-2004 \\
\hline Colombia & 1985-1989 & Colombia & 2000-2004 & Venezuela, RB & 1980-1984 \\
\hline Colombia & 1990-1994 & Costa Rica & 2000-2004 & & \\
\hline Colombia & $2005-2009$ & Rep. Dominicana & $1985-1989$ & & \\
\hline Colombia & 2010-2014 & Rep. Dominicana & 1990-1994 & & \\
\hline Costa Rica & 1985-1989 & Rep. Dominicana & 2000-2004 & & \\
\hline Costa Rica & 1990-1994 & Ecuador & 1980-1984 & & \\
\hline Costa Rica & 1995-1999 & Ecuador & $1985-1989$ & & \\
\hline Costa Rica & $2005-2009$ & Ecuador & 1990-1994 & & \\
\hline Costa Rica & 2010-2014 & Ecuador & 1995-1999 & & \\
\hline Rep. Dominicana & 1980-1984 & El Salvador & $1985-1989$ & & \\
\hline Rep. Dominicana & 1995-1999 & El Salvador & 2000-2004 & & \\
\hline Rep. Dominicana & $2005-2009$ & El Salvador & $2005-2009$ & & \\
\hline Rep. Dominicana & 2010-2014 & El Salvador & 2010-2014 & & \\
\hline Ecuador & 2000-2004 & Guatemala & $1985-1989$ & & \\
\hline Ecuador & $2005-2009$ & Guatemala & $2000-2004$ & & \\
\hline Ecuador & 2010-2014 & Guatemala & $2010-2014$ & & \\
\hline El Salvador & 1990-1994 & Honduras & 1980-1984 & & \\
\hline El Salvador & 1995-1999 & Honduras & 1990-1994 & & \\
\hline Guatemala & 1990-1994 & Honduras & 1995-1999 & & \\
\hline
\end{tabular}


Carlos D. Dabús - Fernando Delbianco - Pablo D. Monterubbianesi

\begin{tabular}{|c|c|c|c|c|c|}
\hline \multicolumn{2}{|c|}{ Alto } & \multicolumn{2}{|c|}{ Medio } & \multicolumn{2}{|c|}{ Bajo } \\
\hline País & Quinquenio & País & Quinquenio & País & Quinquenio \\
\hline Guatemala & 1995-1999 & Honduras & 2010-2014 & & \\
\hline Guatemala & 2005-2009 & México & 1980-1984 & & \\
\hline Honduras & 1985-1989 & México & 1985-1989 & & \\
\hline Honduras & $2000-2004$ & México & 1995-1999 & & \\
\hline Honduras & 2005-2009 & México & 2000-2004 & & \\
\hline México & 1990-1994 & México & 2005-2009 & & \\
\hline Nicaragua & 1995-1999 & México & 2010-2014 & & \\
\hline Nicaragua & 2010-2014 & Nicaragua & 1980-1984 & & \\
\hline Panamá & 1990-1994 & Nicaragua & 2000-2004 & & \\
\hline Panamá & 1995-1999 & Nicaragua & 2005-2009 & & \\
\hline Panamá & 2005-2009 & Panamá & 1980-1984 & & \\
\hline Panamá & 2010-2014 & Panamá & 2000-2004 & & \\
\hline Paraguay & 1980-1984 & Paraguay & 1995-1999 & & \\
\hline Paraguay & 1985-1989 & Paraguay & 2000-2004 & & \\
\hline Paraguay & 1990-1994 & Paraguay & 2005-2009 & & \\
\hline Paraguay & 2010-2014 & Perú & 1980-1984 & & \\
\hline Perú & 2005-2009 & Perú & 1990-1994 & & \\
\hline Perú & 2010-2014 & Perú & 1995-1999 & & \\
\hline Uruguay & 1985-1989 & Perú & 2000-2004 & & \\
\hline Uruguay & 1990-1994 & Uruguay & 1995-1999 & & \\
\hline Uruguay & 2005-2009 & Venezuela & 1985-1989 & & \\
\hline Uruguay & 2010-2014 & Venezuela & 1995-1999 & & \\
\hline Venezuela & 1990-1994 & Venezuela & 2000-2004 & & \\
\hline Venezuela & 2005-2009 & Venezuela & 2010-2014 & & \\
\hline
\end{tabular}

Fuente: elaboración propia 